\title{
Intercambio socio-cultural en una institución de salud
}

\section{Introducción}

El objetivo de la experiencia que se narra fue identificar nuevas prácticas que posibilitaran una intervención más integral en el Centro de Salud «Comunidad Toba» ${ }^{1}$ como resultado de la interrelación entre profesionales y promotores $^{2}$, portadores de los saberes de la cultura de la comunidad. El proceso se desarrolló desde el momento en que la institución promovió reuniones de promotoras, en la búsqueda de un mayor acercamiento con la comunidad.

Este estudio surge de la experiencia en la que participé ${ }^{3}$, desde el año 2001 hasta el 2003, como parte de la práctica curricular de la Licenciatura en Trabajo Social, integrando el equipo de salud que coordinó y planificó las acciones.

Este escrito trata de recuperar el proceso de articulación entre el grupo de promotoras y el equipo de profesionales del Centro de Salud analizando, por un lado, qué prácticas cotidianas del Centro de Salud se transformaron desde las pautas socio-culturales ${ }^{4}$ aportadas por la comunidad toba $y$, por otro lado, qué representaciones se vieron resignificadas en la población barrial, como producto de esta dinámica de relaciones interétnicas.

En principio quiero aclarar que en adelante me refiero a los diferentes participantes del espacio de las reuniones utilizando las siguientes abreviaturas ${ }^{5}$ : Promotoras de Salud (PS), Jefa del Centro de Salud (JCS), Médicos Generalistas (MG), Licenciada en Trabajo Social (Lic. TS), Psicóloga (PSI).

En este artículo, el lector encontrará una síntesis del proceso de la experiencia. Con fines analíticos, se realizarán recortes temporales diferenciados de acuerdo a las temáticas utilizadas, dinámicas de las reuniones y actividades del grupo de promotoras. Considero importante destacar que estos períodos fueron organizados distinguiendo, a su vez, diferentes etapas y ejes problemá-

\footnotetext{
${ }^{1}$ Este centro de Salud depende de la Secretaría de Salud Pública de la Municipalidad de Rosario y se encuentra ubicado en la zona oeste de la Ciudad de Rosario.

${ }^{2}$ El grupo de promotores de salud fue un espacio de reunión organizado por el Centro de Salud, mujeres mayoritariamente, muchas de ellas pertenecientes a la comunidad toba. Durante el trabajo, se encontrará referencia a las mismas como: grupo de promotoras de salud, promotoras, reuniones de promotoras y taller de promotoras.

${ }^{3}$ Agradezco a quienes me alentaron a escribir esta experiencia, especialmente a mi tía Ana G. a Miriam K. y Débora M. como amigas y profesionales, a Nora B. y al Vale por su paciencia.

${ }^{4}$ Se considera importante aclarar que, cuando se nombra lo «socio-cultural» dentro de una determinada cultura, el término «socio» se refiere a la forma de organización familiar y económica particular y el término «cultural» se refiere a las ideas y pensamientos de la comunidad, en este caso, la de los tobas.

${ }^{5}$ Las diferentes abreviaturas en muchos casos serán utilizadas acompañadas por números, los cuales designan a distintas personas, con la intención de preservar la identidad de los entrevistados y/o participantes.
} 
ticos que atraviesan la información recabada durante el proceso de la experiencia: los problemas de comunicación, las relaciones interculturales y el proceso de integración del equipo de trabajo.

Durante el primer período se desarrollan las temáticas de prevención en salud-enfermedad trabajadas y direccionadas por los médicos del equipo. A su vez, se explicita desde qué perspectiva de salud se ha realizado el presente trabajo.

En el segundo período se trabajan resignificaciones socio-culturales de la salud (etnomedicina ${ }^{6}$ ) y de la vida cotidiana de las PS construidas a partir de talleres realizados con esta finalidad. Paralelamente, se expresan algunas categorías y apreciaciones de la comunidad toba relacionadas con la salud, entendiendo que a través de éstas se conocerán algunas generalidades sobre la cultura toba.

En el tercer período se describe cómo las promotoras realizan diferentes capacitaciones y comienzan a ofrecer información, acompañamientos y primeros auxilios a las familias del barrio. Allí lentamente se fue integrando el equipo de trabajo conjunto entre PS y Profesionales.

Finalmente, a modo se cierre, se plantea una serie de conclusiones.

\section{Período 1998-2000}

Las reuniones surgen con el objetivo de crear un nexo entre el Centro de Salud y la comunidad, intentando lograr un contacto lingüístico-cultural con la comunidad toba.

El propósito es que este grupo de promotores funcione como nexo con el resto de la comunidad y que la población del barrio se acerque a la institución a atenderse. Al mismo tiempo, se propuso crear una instancia de capacitación en salud para la población interesada del barrio.

Reconocimiento de las dificultades. El Centro de Salud registra una serie de dificultades centradas básicamente en la comunicación con los pacientes que pertenecen a la comunidad toba, que inciden en la atención de salud. JCS: «Empezamos a principio del '98 porque venía la gente en muy mal estado, con mucho tiempo de evolución de alguna enfermedad, más que nada niños, pero adultos también...y por ahí venían varios de la familia, hablaban entre ellos en toba, no entendíamos nada, preguntábamos y no nos respondían, la cosa era que había una falta de conexión terrible».

Relación con las instituciones barriales. Inicio de la experiencia. JCS: «empezamos yendo a las personas que considerábamos, en ese momento, referentes de la comunidad, a los caciques, pastores, la gente de la vecinal. Entonces, les planteamos que íbamos a hacer un taller de Promotores de Salud y les ofrecimos que podían invitar a toda la gente de su grupo que les interesara. Vinieron más o menos alrededor de 20 mujeres de la comunidad y un varón... Inicialmente no había forma de iniciar una conversación, porque noso-

${ }^{6}$ La etnomedicina nos ayuda a entender los esquemas de representaciones socioculturales de la comunidad relacionados con el proceso salud-enfermedad. 
tros hablábamos y todas las chicas se quedaban escuchando mirando el piso y nadie decía absolutamente nada». MG (1): "Al principio que estaba en el Toba, venían las mujeres, se paraban y no decían nada y yo pensaba, qué les pasará, entonces les preguntaba, ¿te duele ahí? O ¿tenés dolor? iY movían la cabeza!».

En los primeros años se expresan grandes obstáculos para comunicarse tanto en las reuniones, como con las familias de esta comunidad. Las profesionales resaltan las dificultades de comunicación que existen toda vez que están frente a hablantes de una lengua distinta, y que aún en el caso de aquellos que hablan el mismo idioma se presentan igualmente dificultades por pertenecer a una cultura diferente que comprende al mundo de otro modo.

Aquí es importante expresar que desde este escrito se considera a la salud de forma integral, esto significa que el sujeto no puede ser disociado de su historia, su medio social, su relación de deseo, su sentido de enfermedad, su sistema de creencias. Para Laurell «...el proceso salud/enfermedad del grupo adquiere historicidad porque está socialmente determinado. Esto es, para explicarlo no bastan los hechos biológicos sino que es necesario aclarar cómo está articulado en el proceso social» (1982: 16).

Por lo tanto, la dimensión de lo que un conjunto social considera como salud variará de un espacio geográfico, histórico, cultural a otro, es decir, «las enfermedades producen sentidos y significaciones subjetivas y colectivas que operan de diversa manera sobre el proceso salud/enfermedad» (Menéndez, 1991: 103).

Souza Campos, en relación a la forma de intervención de los profesionales, expresa «...es muy importante conocer aspectos genéricos de los procesos salud-enfermedad-atención. Es importante aprender la variación, por esto es importante saber escuchar, y más aún, saber también indagar el caso singular. Y decidir, pero decidir ponderando, escuchando a otros profesionales, exponiendo incertidumbres, compartiendo dudas. Por esto la clínica del sujeto demanda trabajo en equipo y un hacer comunicativo» (2001: 83).

Los Centros de Salud, de Atención Primaria de la Salud (APS), impulsan estrategias para la prevención y la promoción de la salud desde un punto de vista integral, promoviendo la aceptación de la diversidad cultural e ideológica de la sociedad, incluyendo o incorporando el reconocimiento de los saberes de la sociedad. En este escrito se hace hincapié en uno de los pilares básicos en que se asienta la APS, el de promover la participación, que debe ser planteada y entendida como un intercambio permanente de saberes mutuos, entre profesionales y personas de la comunidad.

Contexto de crisis económica. Cuando se presenta la posibilidad de comenzar a recibir los $\mathrm{PEL}^{7}$ y los $\mathrm{PAS}^{8}$ en el Centro de Salud, se plantea que los beneficiarios de estos planes ${ }^{9}$ fueran quienes participaran de las reuniones. Así, se sumó el nuevo objetivo de mantener el espacio de reunión para conservar la continuidad de los planes. Aumenta considerablemente el número de participantes entre 60 y 70 PS.

\footnotetext{
${ }^{7}$ Proyectos de Emergencia Laboral.

${ }^{8}$ Planes de Asistencia Solidaria.

${ }^{9}$ Llamados de esta manera cotidianamente por la gente.
} 
En este primer período, la disposición dentro de las reuniones era: los PS se sentaban en sillas con mesas individuales, en fila, mirando hacia el frente del aula; detrás del escritorio se ubicaban los profesionales del equipo del Centro de Salud. La JCS manifiesta: «al poco tiempo de haber empezado, ya empieza la catástrofe económica y empiezan los programas de emergencia laboral...Bueno, se hace un proyecto y se sortea primero entre las 20 (participantes en las reuniones) 10 (planes), después 10 más y con el paso de los meses había que renovar todos cada tres meses, y cambiar los proyectos. Entonces pensamos con prevención de tuberculosis, prevención de parasitosis, higiene y medio ambiente hasta que llegamos a tener 40 planes, pero en esa época ya teníamos 70 Promotores de Salud, porque la gente venía por las dudas que haya alguna posibilidad, pero le importaba un comino la cuestión de la salud...Era tanta gente, en un momento nosotros ya no sabíamos qué hacer, entonces les propusimos qué les interesaba hacer. Dijeron matemáticas, entonces empezamos a pensar problemas relacionados a la salud, un niño sano debe crecer en el primer año tantos gramos en el $1^{\circ}$ trimestre tanto, en el segundo tantos gramos, en el tercero... ¿Si un chico nace bien y a los 5 meses pesa tanto es un niño sano?».

Los relatos referidos a este período, en su mayoría, expresan las dificultades que dieron inicio a las reuniones. Entre éstas se destacan: los problemas de comunicación; los intentos de establecer vínculos que posibiliten conocer la particularidad cultural de la población para la atención de la salud; la iniciación de una relación intercultural entre la propia visión cultural de los PS y la visión occidental de los profesionales, es decir, las interrelaciones entre estas diferentes formas de comprender la vida cotidiana. A su vez, se destaca en estos relatos cómo la circunstancia de los planes asistenciales da un gran impulso al proyecto, a la vez que desborda las posibilidades de los coordinadores de las reuniones.

\section{Período 2001}

Durante este segundo período asisten de forma fluctuante entre 40 y $60 \mathrm{PS}$, en su mayoría mujeres y unos pocos hombres, por lo cual las temáticas fueron propuestas casi siempre por las mujeres PS.

En este momento se profundizan los objetivos anteriores, incorporándose el propósito de lograr una participación más activa de los PS en las reuniones. La intención que atraviesa el trabajo con las diferentes temáticas fue poder aprehender las resignificaciones culturales que se producían a partir del diálogo con las PS. Además, en algunos casos, efectuar acciones en el barrio que dieran respuestas más pertinentes a esas temáticas, como lo fue Política Ambiental y forestación del barrio. Para esto se determina como objetivo que previamente se planificaran y organizaran las actividades en cuanto a dinámicas y temas de las reuniones, en forma conjunta entre los profesionales que participaban.

Incorporación de profesionales del área social. En el año 2001 se incorporan al Centro de Salud y a la experiencia una Trabajadora Social y una Psicóloga. Con la primera se produce el ingreso de dos estudiantes de Trabajo Social 
como un espacio de práctica pre profesional. MG (1): «...tenemos asignado primero una trabajadora social y después la psicóloga, entonces la lógica cambia, deja de ser desde lo biológico, pasa a ser, sin desatender lo biológico, pasa a tomar otros temas...».

En el relato se plasma la incorporación de nuevos profesionales a la experiencia y al equipo, como una nueva mirada sobre esta realidad en la atención de salud que plantea una resignificación de los temas trabajados en las reuniones.

Cambios en la metodología y los objetivos de las reuniones. La dinámica de las reuniones sufre paulatinamente algunos cambios. Se comienza a trabajar con talleres. Se va generando un importante proceso de comunicación desde el diálogo, el silencio, lo visual, hasta los gestos, entre los PS, mirándose y conversando en voz baja, pausadamente y algunas veces en toba. Se observa también un comienzo de diálogo entre los profesionales que participaban en las reuniones y las promotoras. Es en esta etapa cuando se trabaja con nuevas dinámicas grupales y nuevas temáticas.

La Lic. TS opina: «Además, todo lo que por una cuestión cultural a ellos les cuesta muchísimo hablar sobre ciertas cosas de su cultura o no quieren, digamos, compartirla con otros, bueno, creo que todo ese trabajo sirvió para que cada vez más pudieran ir planteando algunas cosas de su cultura, porque ahí eran explicaciones de algunas actitudes de ellos que tienen que ver con su cuerpo y con las enfermedades».

JCS dice: «a través de ellas entendés una cantidad de cosas, además, a través de ellas, a través de sus propias conductas o sus propias ideas de las cosas, de sus propias formas de hacer las cosas, de sus historias familiares».

MG (1) analiza este momento diciendo: «... se marca mucho cuando se deja de hablar de lo biológico y cambia lo que se trabajaba...Bueno, cuando aparece el tema de pasar películas, el tema del maltrato, se corrieron (los temas biológicos) porque aparece una lógica de trabajo diferente. Claro, ahí es donde yo vi que se horizontalizó, o sea, donde ellas, las mujeres, podían conocernos también a nosotros y nosotros a ellas, sus historias y dejar un poco nuestro lugar de médicos. Y yo creo que ahí fue donde se dio el intercambio». .

Intercambio de saberes. Aparece en las reuniones la posibilidad de que se expresen las representaciones socio-culturales que tiene la comunidad acerca de la salud y otros aspectos que hacen a la vida en general. Asímismo, las PS plantean inquietudes sobre la medicina blanca.

Los temas que se abordaron son: salud de la mujer, planificación familiar en la cultura toba y la que ofrece el Centro de Salud. Las PS decían: «la mujer debe estar en buen estado físico para cuidar a sus hijos y seguir adelante en el hogar, tiene que saber las enfermedades para cuidar y prevenir a su familia y a mí». "¿Cómo detectar y eliminar el cáncer de mama?, ¿y el de útero? ¿Se tienen que dejar de tener relaciones sexuales después de la menopausia? ¿Cuáles son las consecuencias de las pastillas anticonceptivas?». Todas estas cuestiones son trabajadas durante las reuniones.

En los registros de las reuniones mencionadas es posible visualizar la forma como piensan su salud reproductiva.

En uno de los encuentros las PS transmiten cómo se cuidan, explican que 
toman remedios caseros y cómo usan el palo santo (cosakait), que lo utilizan en forma de infusión: «eso me mantiene si mi nene tiene ya 3 años»; "yo tengo relaciones con mi marido mientras menstrúo solamente»; "El hombre sabe...hombre está bien si se cuida con remedios caseros..., en el Centro de Salud no diu, pastilla»

A su vez, aparecen «temores de píldoras anticonceptivas»; «hay vergüenza de los ginecólogos hombres».

En los planteos realizados por la PS aparece claramente la convivencia de dos visiones de la vida cotidiana y sus influencias relacionadas a la salud, desde las representaciones sociales tradicionales de la comunidad toba y desde una visión médico-cientificista. En charlas posteriores a la proyección de la película «Como agua para chocolate», surgieron historias familiares y de la infancia de muchas de las PS con referencia a los valores que animan la vida cotidiana: «antes cuando eran niñas vivían en la esclavitud». Además, historias de vidas en el Chaco y en Rosario: «Debían levantarse a las 4 de la mañana para ir a cosechar...trabajaban todo el día, hasta que caía el sol». En el Chaco, «todos trabajan, chicos y grandes, por eso muchas mujeres no saben leer $y$ escribir». Acá en la cuidad "se changuea», "salen a pedir... cuidan autos...venden artesanías...cirujeo...venden cartón y botellas»; "Acá hay diferentes cosas para sobrevivir».

Con el tema de los jóvenes del barrio, dicen: «los fines de semana toda la familia se va al centro... hay veces que hay que ayudar a los padres»; «muchos jóvenes buscan trabajo y no lo encuentran, se deprimen, salen a robar...por no conseguir trabajo, buscan eso, la bolsita». "Hay muchos padres que los zamarrean y así no se puede hacer nada...hay que hablar».

Todos estos planteos surgidos sobre sus historias de vida y su cotidianeidad son importantes de analizar, considerando que influyen en la historia particular de los sujetos, revelando ciertas cuestiones sobre la organización socio-cultural dentro de las tradiciones y costumbres tobas. Así, los relatos de las historias de vida en el Chaco y en Rosario asumen gran valor para poder trabajar en la atención de la salud desde una mirada integral.

Aquí se abrirá un paréntesis para expresar consideraciones sobre lo cultural, que nutrirán la posibilidad de análisis del texto. La cultura es considerada por Tylor como «un complejo que abarca conocimientos, ideas religiosas, artes, costumbres, derecho, uso y toda aquella gama de capacidades que adquiere el individuo como miembro de la sociedad.» (apud Di Tella, 2001: 153), dentro de ésta, se hallan las representaciones sociales que son parte de las interacciones del grupo.

Las representaciones sociales son definidas como «construcciones simbólicas, productoras de sentido que contienen una explicación y/o interpretación del mundo y actúan en la organización de las prácticas sociales» (Carracedo et alli, 1996: 26-27).

A partir de aquí realizaremos un breve recorrido sobre las representaciones y apreciaciones de la comunidad toba relacionadas a la salud (etnomedicina). «Desde la perspectiva $q o m^{10}$, la salud depende del equilibrio entre las relacio-

${ }^{10}$ Qom: El término qom o kom es el autoetnómino, es decir, cómo el grupo se autodenomina; significa «gente en el sentido de nosotros». Los españoles y sus enemigos los llamaron tobas, que significa frentones, por la costumbre de raparse el pelo de la frente en señal de duelo, éste es el exoetnómino (nombre dado por los demás). 
nes entre los hombres y entre éstos, la naturaleza y lo sobrenatural. Consecuentemente, la enfermedad se considera causada por la trasgresión de reglas que deben ser respetadas o por daños realizados por los brujos que producen alteraciones en dicho equilibrio» (Vázquez et alli, 1998: 259-260).

Es decir que las causas de las enfermedades se atribuyen a la ruptura de reglas (involuntaria o voluntariamente), envío de daño mágico por parte de un piogonak o un brujo. La etnomedicina nos ayuda a entender los esquemas de representaciones socioculturales de la comunidad toba relacionados con el proceso salud-enfermedad. Los piagonak o chamanes son quienes se ocupan de preservar la armonía entre los hombres y los dioses teniendo poderes curativos, mágicos y adivinatorios. La forma de curar de estos es chupando o soplando donde se encuentra el mal. Los piagonak o los chamanes son valorizados por su saber y la rapidez de los diagnósticos y curaciones. Los tobas utilizan ciertas hierbas y yuyos para cuidados medicinales. Se reivindica la afirmación de Vazquez et alli cuando dicen que: «la población del barrio en general recurre alternativamente a Shamanes, curanderos, pastores y médicos para la cura de distintas enfermedades. Las variantes de sexo, edad, tiempo de permanencia en la ciudad y lugar de procedencia implican diferentes representaciones sociales y prácticas sobre el proceso salud/enfermedad...La demora en los diagnósticos, eso como los interrogatorios efectuados por los médicos (no usuales en la cura Shamánica) suponen, desde la perspectiva toba, ignorancia de la enfermedad, lo que contribuye, conjuntamente con las barreras comunicacionales, a desconfiar de la eficacia de la medicina científica. Los menores de 30 años, sobre todo las madres, utilizan en lo posible los servicios que ofrece el dispensario barrial o los hospitales públicos» (1998: 260).

Cuando la enfermedad es considerada grave, se vuelve al «monte» para tratarse con los curadores indígenas de mayor prestigio, o se vuelve al Chaco en busca de yerbas medicinales. La organización familiar es extensiva en donde dos o tres generaciones conviven en la misma casa. La mujer toba ocupa un lugar de privilegio dentro del grupo, aparece claramente marcada la jerarquía por edades, las mujeres de mayor edad son las más respetadas y consultadas; cumplen un papel central en la transmisión de saberes, proceso de endoculturación, lengua qom, ella es quien la trasmite a sus hijos, junto con los mitos y las historias de «los antiguos» (Viglianchino, 1998: 71). Como señalan Carracedo et alli, «su saber está relacionado con los comportamientos preventivos en salud y enfermedad» (1996: 27). Además, juegan un papel muy importante en los procesos de «control» social intraétnico, mantienen el poder en los procesos de embarazo, parto y lactancia; «...durante el embarazo, parto y lactancia, la mujer toba debe respetar una serie de reglas, algunas relacionadas con la alimentación, y otras con la conducta en general. La ruptura de ciertas reglas dañaría fundamentalmente al bebé, y también a la familia» (Carracedo et alli, 1996: 31).

Quienes vigilan a las madres para que cumplan las reglas son las mujeres ancianas, como por ejemplo: «durante embarazo y lactancia las relaciones sexuales están prohibidas. Si la madre ha tenido relaciones en el embarazo, el bebé nace sucio, verdoso y puede presentar malformaciones. En el caso que la madre tenga durante el amamantamiento relaciones sexuales (...) la criatura se arruina, la carne se le afloja, no crece. Las abuelas le dicen a la madre: 'no 
querés a tu hijo'»; «las mujeres más grandes se dan cuenta cuando tenés un 'chonec'»'11. Otra de las reglas, relacionadas con la alimentación es que durante el embarazo la mujer no puede comer el estómago de un animal o ningún animal muerto.

En este período que aparecen las cuestiones socio-culturales se explicitan, por ejemplo, temores y descreimiento sobre lo que plantea la salud occidental en cuestiones ginecológicas o de planificación familiar. Por otro lado, el lugar de privilegio que la mujer ocupa en la comunidad relacionado a los comportamientos sobre control de salud y enfermedad o la creencia del chonec que se puede interpretar como una explicación de la comunidad frente a los chicos desnutridos, con decaimiento, etc., se considera de mucho aporte para poder comprender por qué no se acercan al Centro de Salud o minimizan la situación omitiendo las recomendaciones de los profesionales.

Todas estas situaciones deben ser resignificadas considerando el concepto de proceso salud-enfermedad de Laurell y Menéndez expresado anteriormente y las propias consideraciones y representaciones de la comunidad toba relacionadas con la salud, posibilitando el replanteo de los diversos enfoques.

A partir de todos estos conocimientos, tradiciones y creencias transmitidos por las promotoras, los profesionales que participan de las reuniones comienzan a tener algunos conocimientos de la cultura de la comunidad toba. Pero, paralelamente, continuan las dificultades de comunicación referidas a los obstáculos que se presentaban a los profesionales para poder diagnosticar e intervenir y para llevar adelante los tratamientos.

La JCS expresa: «Sí, tiene que ver con la atención, pero por lo menos como para tener una visión más amplia en el momento de hacer un diagnóstico, porque vos por ejemplo preguntás a la mamá si el paciente tiene fiebre y te dicen 'sí, cuando le agarra el ataque de tos, sí tiene fiebre'. Es decir, tienen una forma de relatar las cosas que vos tenés que tomar en consideración para poder, en total, con la clínica y todo hacer un diagnóstico».

Otro ejemplo, dice Lic. TS: «El tema de la diferencia de la interpretación del tiempo y que por ahí es muy difícil interpretar, si pasó algo hace 1 día, 2 días o una semana o cuándo...El mucho o poco tiempo es diferente el de ellos al nuestro».

MG (1): «las mujeres por ahí venían con el yuyo que se hacían ver por el piogonac. Yo les decía que se cuiden con el té, pero que también tomen las pastillitas (risa)...yo venía con todo esto de que vos tenés un hijo y empezás a tomar pastillas. Yo lo repetía, lo repetía, yo decía ¿te vas a cuidar? y no me daban ninguna importancia, hasta que un día pregunté ¿y vos cuándo empezas a tener relaciones? Yo empiezo a tener relaciones después que el pibe tiene un año!. O sea, no tiene nada que ver. Si yo culturalmente lo sabía le decía, mirá, cuando te definas a empezar a tener relaciones, antes avisame así vemos $y$ empezás a tomar pastilla, pero no así encajarle».

El proceso por el cual transita la experiencia, durante este período, puede ser analizado como de gran ampliación de la comunicación durante las reuniones entre profesionales y PS. Pero donde persisten grandes dificultades de

${ }^{11}$ Denominación dada al niño que presenta debilidades físicas, razón que se atribuye a que su madre tuvo relaciones sexuales durante embarazo y parto. 
comunicación es entre las personas de la comunidad Toba que se atienden en el Centro de Salud y los profesionales del equipo, es decir, permanecen las dificultades de comprensión e interpretación dentro de los consultorios o en las intervenciones particulares. Así, por ejemplo, los conocimientos incorporados en relación a tradiciones, costumbres, mitos, hábitos cotidianos, sus historias de vida y sus valores comunitarios pueden ser resignificados y comprendidos como parte de este proceso de conocimiento de la particularidad de las familias de la comunidad, lo cual significa un gran acercamiento a una mirada más integral para la intervención en la salud, en cuanto a las características generales de la comunidad, pero continúan las dificultades en las situaciones particulares (a intervenir como profesionales de la salud).

Es decir, se expresa la convivencia de dos visiones de la vida cotidiana (interrelaciones culturales) y sus influencias relacionadas a la salud, desde las representaciones sociales tradicionales de la comunidad toba y desde una visión médico cientificista (proceso salud-enfermedad).

\section{Período 2002-2003}

Sumado a los objetivos de los períodos anteriores, paulatinamente se pretende, mediante un trabajo en conjunto, que los promotores sean portavoces de la comunidad, de las necesidades y de las condiciones de vida del barrio. Se plantea también como objetivo que los PS asistan, dentro de sus posibilidades, a las familias del barrio, ofreciendo información, acompañamientos y primeros auxilios, pero estableciéndose claramente que no reemplazarían a los médicos, ni al resto de los profesionales. Se pretende, asimismo, repensar desde los saberes culturales de las promotoras las diferentes dificultades que se presentaban en el Centro de Salud para asistir a las familias de la comunidad.

A principios de 2002 el grupo comienza a funcionar en el Crecer $N^{\circ} 22$. Allí se dispone de equipo de mate, hecho que incide en que se genere en los encuentros un mejor clima y, por lo tanto, mayor fluidez en el debate. En el Crecer se dispone de mesas grandes rectangulares, lo que induce a que se integren en 2 o 3 grupos, los PS con los profesionales. A mediados de ese mismo año, se accede a un nuevo sitio que había sido utilizado por la municipalidad de Rosario como Obrador.

Cuando las reuniones son en el Obrador, el grupo va adoptando una nueva distribución espacial y se integra en una ronda, sin lugares fijos y de modo que todos se ven de frente con todos. Las PS comienzan a llevar el mate que circula en la rueda, posibilitando y favoreciendo la circulación de la palabra en los encuentros.

Afianzamiento del grupo de Promotoras de Salud. Las Promotoras comienzan a inscribirse en los planes Jefas/es de Hogar. La modalidad de estos nuevos planes hace que se reduzca el grupo de promotoras (entre 20 y 30) y sólo permanezcan aquellas a quienes más les interesan las reuniones y actividades que se realizan.

El grupo se plantea modos de llegar a otras familias del barrio buscando la posibilidad de capacitarse en primeros auxilios, Curso de Lactancia Materna, 
Programa de Boor, broncoespasmos de niños ${ }^{12}$, censo y relevamiento de vacunas para ampliar la acción del Centro. Se afrontan dificultades de comunicación en hospitales y en otras dependencias públicas, la importancia de la privacidad de las familias y diversas alternativas a situaciones críticas cotidianas y urgencias.

La PS (1): «nosotros conocimos que en otros Centros de Salud no lo hacían, que no lo hacían esto del seguimiento y acompañamiento de personas... Yo sé esto que por ahí en otros Centros de Salud no es necesario, mucho, como en la gente de la comunidad, que va y viene del norte, que no puede, no sabe tomar los colectivos, todas esas cosas, o que tiene directamente otro idioma, otra lengua, digamos, y para esto es importante, acompañarlos y todo».

Es importante destacar el relato de la PS (1) donde ella resalta la importancia de su trabajo como PS dentro de la particularidad cultural del barrio y este contexto particular.

Experiencias de trabajo conjunto entre profesionales y promotoras. En la medida en que las PS presentan problemas que se daban en el barrio y los profesionales hacen preguntas sobre las representaciones de la comunidad toba acerca de los temas abordados, se debaten formas de intervención conjunta. Se presentan dificultades para conocer nuevas familias del barrio, y los problemas que se presentan en él o, cuando no estaba abierto el Centro de Salud, aquí surgen las diversas tareas de colaboración realizadas por las PS; tareas en las que se evalúan los resultados logrados, el grupo crece, se fortalece y encuentra rumbo propio. Todas estas actividades realizadas por las PS son coordinadas, acompañadas y supervisadas por la Lic.TS, por médicos, la PSC y las ETS.

La PS (2) dice, sobre la experiencia de acompañamientos a hospitales: «Era una señora que tenía, ¿puede ser un quiste? en el ovario, como principio de cáncer, tenía que llevarle todas las semanas dos veces por semana, al (hospital) Clemente Álvarez, con el cirujano, con la ginecóloga, porque ella no le entendía a los médicos y la hija no le acompañaba!. Yo la acompañaba, le llevaba a todos lados, a todos los hospitales, le sacaba los turnos, decí que todos los médicos eran buenos, y me daban lo turnos programados, si no yo me tenía que ir a las 6 o a las 5 de la mañana a sacar el turno. Para internarla yo le dije a la hija, mirá tenés que acompañarla para internarla mañana a las 10 de la mañana, y del hospital me llamaron porque decían que 'no le entendían a la hija. Así que me tuve que ir yo y ahí cuando me vio el doctor me dijo 'ah, sí, sos vos...sí, ella está para internación'. La hija me dijo 'ella no quería saber nada de estar conmigo, ella me dijo, llámale a la PS (2), que ella sabe todo lo que yo tengo'. Ella entendía castellano, por ejemplo, yo le hablaba castellano, continuamente ella me hablaba en toba y yo en castellano. El doctor explicaba y ella no le entendía, pero entonces el doctor me preguntaba y yo le decía ella no le va a entender y él me explicaba y yo después venía y le explicaba a la hija para que ella le explicara bien, porque yo no sabía cómo explicarle. Y después la operaron, todo bien y después ya se encargó la hija, si yo le dije al doctor 'se

\footnotetext{
${ }^{12}$ Luego del curso de primeros auxilios y el programa de Boor, las PS comenzaron a tener botiquines con varios nebulizadores en sus domicilios para utilizar durante los fines de semana y en los horarios en que el Centro de Salud estaba cerrado.
} 
tiene que encargar la hija' y aparte la psicóloga acá le dijo, 'ella es tu mamá y vos tenés que encargarte' y la Lic. TS también, 'ya PS (2). hizo el camino, le acompañó, le llevó, le consiguió todo, ahora es tu responsabilidad ayudarla'».

En esta experiencia se da un acompañamiento a raíz de dificultades de la población para manejarse en instituciones de salud, en este caso, de mayor nivel de complejidad. Se puede observar en esta circunstancia particular relatada, «cierta accesibilidad brindada desde el sistema de salud» a esta situación de la población atendida. Por otro lado, se puede analizar la seguridad que brinda a esta persona el acompañamiento de la PS, quien conoce su cultura y lengua particular y quien sabe su situación de salud. Además, la importancia que reviste en la señora el vínculo de la PS con los profesionales médicos del Hospital. Haciéndose explicita la orientación brindada por parte de la PSI y la Lic. TS. del Centro de Salud, resaltando la responsabilidad ineludible de la familia.

En este trabajo conjunto de los profesionales con las PS se hace una resignificación de los principios básicos de la APS, de promoción de la participación y lo expresado por Chiaradía que plantea se «debe trabajar en conjunto con este agente (promotoras de salud o agentes sanitarios), portavoz de la comunidad, guiando, supervisando su trabajo, sosteniendo por instrucciones precisas así como por suministros regulares. Es decir descentralización-centralización efectiva, ida y vuelta de la información, desde este agente de salud al equipo polivalente del centro, de éste al hospital, de ahí a los organismos centralizadores del sistema de salud, es decir crear la red de la que hablamos. Si no se da así no se puede hablar de atención integral, continua, accesible, aceptable y tampoco se puede hablar de cambio, transformación» (Chiaradía, 1992: 8).

En este último relato continúan presentes las dificultades de comunicación y las interrelaciones interculturales, entre la visión de la población toba, las PS y diferentes profesionales, ya sean del Centro de Salud o de otras instituciones de salud de mayor nivel de complejidad. Y es en la problematización de estas cuestiones que se va creando un proceso de integración del equipo de trabajo entre profesionales y PS, apostando al trabajo conjunto desde las diferentes miradas. Las PS brindan aportes muy valiosos al jugar de intérpretes entre pacientes y médicos y viceversa, tanto en lo referente a lo estrictamente lingüístico (traducciones) como a las diferencias que surgen por pautas culturales diversas (costumbres y temores de la comunidad). Al establecerse vínculos entre los profesionales y las PS se plantea que desde ambas partes se conocieron y se incorporaron saberes que son muy importantes. Del lado de los profesionales, para poder intervenir y llegar mejor a la gente durante la atención cotidiana en el Centro de Salud, lo cual no implica que desaparezcan las contradicciones entre ambas formas de comprender la salud.

La Lic. TS recuerda: «Para las instituciones, por ejemplo, fue importante cuando acompañaban a la hospitales, en la Maternidad o CEMAR, porque también creo que dejaron marcas en esas instituciones, porque hasta el día de hoy a veces llaman diciendo, puede venir algún Promotor de Salud por esta situación, o sea que fue importante, porque me parece que abrían canales de acceso, bueno, esto de que alguien pueda ser intérprete en las cuestiones del idioma o, bueno, interpretar lo que se está hablando para una familia y fue importante para la propia institución también». 
La MG (1) desde su experiencia dice: «Cuando las promotoras traían pacientes, eran embarazadas complicadas, tuberculosos complicadas, todo eso, era grave, no venían por una fiebre así no más, tenían esa capacidad ellas de pescar y decir ésto lo tienen que ver o tiene que llegar al Centro de Salud, un chico desnutrido o, yo creo que pudieron tener ese ojo diferente, esa mirada diferente, con esos conocimientos diferentes que habían adquirido. Ellas venían y describían cuál era la situación, porque ellas los controlaban con lo que sabían y ahí uno decía adónde está vamos a buscarlo».

Se hace totalmente explícito el juego de interrelación intercultural existente entre ambas visiones las cuales implican costumbres, tradiciones, mitos y temores que inciden en la probabilidad de intervención en la salud, contemplando la particularidad de la población. Las recopilaciones de entrevistas a profesionales expresan sus conocimientos sobre cuestiones culturales, ideas, valores, temores, concepciones y tradiciones que atraviesan la posibilidad de intervención en la salud y la importancia que estos temas culturales sean resignificados y considerados para el trabajo conjunto.

PS (3) dice: «En una época, tuvimos una emergencia que una señora tuvo un parto en el domicilio, así que PS (2) y yo, llamamos por teléfono (al SIES), y como yo sabía cómo estaba, de cuántas semanas y todo eso, entonces le leí cómo estaba, le digo yo: 'está a término porque está de 37 semanas y media' y dice '¡Ah, bueno!' Y él me dice '¿Ustedes qué son?', 'yo me llamo PS (3), soy Promotora de Salud, trabajo en el Centro de Salud Toba, hago traducción y esta señora está en el domicilio de pasaje 1819, nosotros los vamos a esperar ahí!'. Mi papá en esa época veía y entonces con la linterna hacía señas y le hacía señas para que se fueran más adelante el móvil de la ambulancia».

Sobre la misma situación, la JCS cuenta: «...un fin de semana que la llamaron a PS (3) y a PS (2) acá en la Tacuarita, por un parto. Ellas fueron, Ilamaron al SIES, llegó cuando el chico ya había nacido, así que ellas estuvieron en el parto. Después estuvieron atentas a cuando volviera y cuando vuelve...viste que esta cuestión de después de parir las mujeres por 40 días no pueden salir de su casa porque se exponen a riesgos y ellas lo veían muy mal al bebé y lo convencieron al marido de que lo traiga, y lo iban a buscar todos los días, tuvimos que volver a mandarlo a la Martin porque no aumentaba. Después de esa internación, seguían yéndolo a buscar para venir a controlarlo y una vez PS (3) se queda hablando con la madre y se da cuenta que la mujer a la noche no le daba la teta, por qué no lo sabemos, pero no le daba la teta, y entonces le explicó que también le tenía que dar a la noche y qué sé yo...y entonces el bebé empezó a aumentar».

La PS (1) comenta: «Me acuerdo un caso que dijo el doctor que si yo no la hubiese acompañado al hospital Vilela a tiempo a una mamá con un nene, podría haber causado... dijo 'la verdad que el trabajo que hacen las promotoras, es importantísimo'. Y que uno se haya dado cuenta, aunque la mamá no se ha dado cuenta, le había agarrado como un tipo neumonía, que yo me di cuenta porque la doctora me había contado cómo arrancaba, con agitación, y lo controlé».

JCS cuenta:»...después empezaron a llamar de la Martin cuando había alguna chica que había ido a tener su bebé allá y tenía algún problema...Mirá con esta chica de la colostomia, que la mamá se pasaba todo el tiempo llorando, fue PS (3) a verla, porque Ilamaron de la Martin diciendo que por favor vaya 
una Promotora de Salud, fue PS (3) a verla y resulta que ella pensaba que esto le iba a quedar para toda la vida, que el intestino saliéndole por el abdomen...iqué horror! .Entonces ella habla con los médicos, le explicó y la mujer entendió y listo».

Sobre la misma experiencia, la PS (3) señala su vivencia: «...de traducción yo fui con una mamá que tenía que llevar a una bebé, el primer paso yo fui a verla al hospital a ver qué pasaba, y después siguieron otras promotoras, ella era como que le tenía miedo a la hija que había nacido con un problemita. Después le pregunté y ella dijo 'que no, a los otros hijos haber nacido normal, el verla así tenía miedo de lastimarla'. Pero de que ella le daba el pecho y todo eso sí le daba, pero lo que pasaba es que ella delante de los médicos no le daba, pero siempre se quedaba con hambre. Después igual empezaron a darle complemento».

Es importante resignificar la apropiación de las promotoras de saberes transmitidos por los profesionales del Centro de Salud en estas capacitaciones, en cuanto a esas mínimas prácticas de atención durante los fines de semana, posibilitando resolver o facilitando algunas cuestiones en las urgencias, por ejemplo. Produciéndose en el seno de la comunidad toba pequeños acercamientos a la medicina oficial. Es preciso destacar que los conocimientos incorporados por las PS son utilizados en su vida cotidiana, lo cual no significa que como miembros de la comunidad toba dejen de utilizar sus saberes tradicionales de la medicina toba.

Mayor capacitación. Priorización de criterios en las acciones de las PS. A medida que el proceso de capacitación a través de las reuniones semanales va avanzando, las PS adquieren una serie de conocimientos, criterios para actuar, para adecuar su discurso preservando incluso cierta intimidad de sus pares del barrio. En particular, en lo relacionado con la defensa de su propia cultura y haciendo cada vez más efectivo su trabajo.

PS (3): «O sea, tratar de enseñar a respetar la cultura, el saber de la comunidad. Decir, se dicen muchas cosas, pero desde lo profundo del saber no se puede, sólo dentro de la comunidad. Si yo sé algo tengo que transmitir a mi hijo o a mi sobrinos y ahí termina, porque es un saber que pertenece a un grupo, eso se está perdiendo».

PS (1) dice: «trabajar en las urgencias, por ahí me identifico como promotora cuando te piden un dato específico. Por ahí nos preguntan ¿y usted cómo sabe? $Y$ ahí sí digo 'yo soy PS y trabajo con el Centro de Salud'. Yo siempre que iba a acompañar me presentaba como promotora, que vivía también en el barrio, y le comentaba por qué necesidad se iba esa persona. $Y$ yo siempre traté de que la gente tenga la intimidad del doctor en cada paciente».

PS (2) observa: «La PS (6) la semana pasada me dijo que estaba muy mal, que tenía mucha fiebre, le dolía la cabeza, le dolía todo el cuerpo, que vino acá, le pusieron una inyección, le dieron pastillas, dice, pero no le hacía nada... Mi marido se fue a buscar a no sé quién, y me sacó un montón de cosas de acá (realiza gesto: se toca el pecho de lado a lado de su cuerpo) y era eso, no más que me estaba doliendo', entonces le dije yo 'alguien te tiró algo', 'sí', dijo ella, 'alguien me tiró algo'. También está el problema de los chicos muy rebeldes, por esa causa. La placenta de él yo qué sé dónde está, estará en el basural o comió algún perro o algo, y yo qué sé cómo va a ser el día de mañana mi hijo». 
Al final del 2003 las PS plantean la necesidad de redefinir su rol en el barrio, su responsabilidad personal y las que pertenecían a las familias y a la comunidad, ya que algunos punteros políticos, vecinos o líderes, las hacen cargo de todo lo que tiene que ver con la salud del barrio.

En esa reflexión llegan a las siguientes definiciones sobre su trabajo: «el diagnóstico no lo dan los Promotores de Salud, lo dan los médicos», «las promotoras no son médicos sino primeros auxilios», «las promotoras no medicamos», "los responsables del paciente son los familiares», «las promotoras tenemos familia y responsabilidades», «queremos que nos respeten en nuestro trabajo, no somos responsables de todo, no tenemos autoridad dentro del Centro de Salud». Con relación al tema, la PS (1), expresa: «Algunos requieren más de las promotoras, exigen más, pretenden siempre más!!».

Luego de todo lo expuesto hasta aquí, se puede conocer el proceso por el cual fue transitando la experiencia hasta llegar a la conformación del equipo de trabajo. Las capacitaciones, en cuanto a técnicas de la salud occidental y criterios para trabajar, hicieron al fortalecimiento del trabajo conjunto, manteniendo de forma constante un lugar de importancia a las interrelaciones interculturales que atraviesan el proceso.

\section{Conclusiones}

Considerando que el objetivo general planteado al iniciar este trabajo era identificar nuevas prácticas que posibiliten una intervención más integral en la salud, como resultado de la interacción entre profesionales y PS, puede señalarse que el proceso que se dio en esta experiencia de trabajo conjunto en el Centro de Salud, así como las prácticas que se generaron a partir del mismo, fueron novedosas y exitosas en muchos aspectos. Como se ha podido apreciar a través de los testimonios, la participación activa de las personas del barrio, que brindaron sus conocimientos y formas de comprender la vida desde su cosmovisión y desde la cultura de la comunidad a la que pertenecen, aportaron a los profesionales de la salud invalorables herramientas para la comprensión, intervención y diálogo.

Las modificaciones que se presentaron en las prácticas de atención de salud se expresaron fundamentalmente en las diferentes estrategias de intervención llevadas adelante y que, según la opinión de los diferentes actores entrevistados, resultaron exitosas. El proceso que nos propusimos describir registra los avances que van desde aquellas primeras reuniones en las que las PS mantenían un rol pasivo y el grupo no encontraba caminos efectivos para el diálogo, hasta llegar a la comunicación fluida y la incorporación a la dinámica del Centro de Salud.

Las promotoras hicieron de nexo entre todos los actores involucrados en la atención de salud, colaborando, desde su rol, tanto en la comunicación, comprensión e interpretación con los profesionales del Centro de Salud como con los de otras instituciones y con las personas atendidas y sus familias.

Fue de fundamental importancia la supervisión y coordinación de las tareas por parte de los profesionales que las organizaban (JCS; MG; PSI y Lic. TS). Además, es central destacar que las prácticas cotidianas de atención de salud se ven transformadas cuando se incorpora la dimensión socio-cultural en los 
profesionales del equipo del Centro de Salud, como vemos en algunos párrafos de las entrevistas a JCS y MG (1). Estos cambios se dieron en quienes coordinaron el trabajo con las promotoras, es decir, quienes tuvieron un vínculo fluido en el trabajo conjunto o se involucraron personalmente en conocer las diferencias culturales que incidían en su acción profesional.

Además, para esta experiencia, se considera de suma importancia el trabajo en equipos interdisciplinarios, que brindan diferentes miradas para comprender una realidad tan diversa como la que en este caso se ha dado. La experiencia caló hondo en todos los actores involucrados, lo que se evidencia en los recuerdos que se expresaron en las entrevistas, para las que no hubieron trabas y donde coinciden -a pesar del tiempo transcurrido ${ }^{13}$ - numerosos detalles señalados en los testimonios. Todas las pautas culturales y representaciones sociales comunitarias aportadas debieran ser conocidas y consideradas por quienes intervienen en este barrio o en las diferentes instituciones comunitarias.

Por todo lo expresado con relación a la particularidad de la comunidad toba, es fundamental que este trabajo conjunto con la población barrial sea realizado de forma estable en instituciones insertas en un contexto de amplia diversidad cultural. La articulación intercultural debería convertirse en una política de Estado con los recursos económicos y humanos específicos, necesarios para la construcción permanente de una atención de salud que apunte fuertemente hacia una mirada más integral de los sujetos.

Es necesario destacar la gran deuda que el sistema educativo tiene con los egresados de casi todas sus instituciones, con respecto a la realidad multicultural y multilingüe argentina. Los profesionales de este Centro de Salud señalan su asombro y desconcierto en los primeros contactos con estos argentinos distintos y cómo debieron buscar por sus propios medios allanar los caminos para relacionarse con ellos. El diálogo con los otros culturalmente distintos fue posible gracias al compromiso humano y social de los involucrados en la experiencia.

${ }^{13}$ Transcurrieron cuatro años desde el 2003, de la experiencia, hasta el 2007, momento en que se realizaron las entrevistas. 


\section{Bibliografía}

ACHILLI, E. SANCHEZ, S. «La vida social de los tobas», en: Secretaría de Salud Pública. Rosario, Argentina, Ediciones AMSAFE, 1997.

BASSET, E. «Exclusión, ciudadanía y salud», en revista: Salud, problema y debate. Rosario, 1998.

BELMARTINO, S. «Nuevas reglas de juego en los servicios de salud», en revista: Salud, problema y debate. Rosario, 1998.

BIGOT, M. RODRIGUEZ,G. VAZQUEZ, H. «Los asentamientos tobas en la ciudad de Rosario». en: RADOVICH, J. BALAZOTE, A. La problemática indígena. Estudio antropológico sobre los pueblos indígenas de la Argentina. Rosario, Centro Editor de América Latina, 1992.

CARRACEDO E. «Representaciones y prácticas sobre los procesos de salud y enfermedad infantil de un grupo de mujeres tobas», en: Revista de la escuela de antropología. Vol. III, Rosario, Facultad de Humanidades y Artes. UNR, 1995.

CARRACEDO, E. «Migraciones, situación de salud y tuberculosis en grupos tobas de la ciudad de Rosario», en: Papeles de trabajo $N^{\circ} 7$. Rosario, Centro Interdisciplinario de Ciencias Etnolingüísticas, UNR, Octubre 1998.

CARRACEDO, E y VIGLIANCHINO M. «Control social y representaciones sociales sobre salud y familia en un grupo de migrantes tobas», en: Papeles de Trabajo $N^{\circ} 5$. Rosario, Centro Interdisciplinario de Etnolingüística, UNR, 1996.

CARBALLEDA, A. La intervención en lo social. Exclusión e integración en los nuevos escenarios sociales. Buenos Aires, Paidos, 2002.

CHIARADÍA, L. «Atención Primaria de la Salud». Mimeo Cátedra, s/l,1992.

CZARNY,G. «Sitios para la identidad», en: Revista de la escuela de Antropología. Vol. II, Rosario, Argentina, UNR, 1994.

DI TELLA, Torcuato y otros. Diccionario de ciencias sociales y políticas. Facultad de Humanidades y Artes, Buenos Aires, Ediciones Emecé, 2001.

FERNANDEZ M. R. y HACHÉN R.R. «El rol de la mujer qom (toba) como agente de endoculturacion: estrategias para una interacción cultural», en: Papeles de trabajo $N^{\circ} 4$. Rosario, Centro interdisciplinario de ciencias etnolingüísticas y antropológico-sociales, Facultad de Humanidades y Artes, 2005.

GARBULSKY, E; ACHILLI, E; SANCHEZ, S; «Los tobas en Rosario. La lucha por los derechos en la ciudad del desempleo y la marginación», en: Revista de la Escuela de Antropología. vol. 5, Rosario, UNR, noviembre de 2000.

LAURELL, A. «La salud como proceso social», en: Revista CESS. n. 19, 1982.

MENÉNDEZ, E. Morir de alcohol. México, Alianza Editorial, 1991.

PARSONS, T. «El sistema social», en: JIMÉNEZ BLANCO, J. y CAZORLO PÉREZ, J. Ediciones de la Revista de Occidente, Madrid, 1966. 
PENNA, M. «Control social y representaciones sociales sobre salud y familia en un grupo de migrantes tobas», en: Papeles de trabajo $N^{\circ} 5$. Rosario, Centro Interdisciplinario de Ciencias Etnolingüísticas, UNR,1996.

SALLERAS SANMARTÍ, L. «La salud y sus determinantes», en: Revista Anthropos. España, n. 118-119, 1991.

SOUZA CAMPOS, G.W. Gestión en salud. En defensa de la vida. Buenos Aires, Editorial Lugar, 2001.

VAZQUEZ, H. BIGOT, M. «Contacto linguistico-cultural, sincretismo, y funcionalidad de la lengua nativa entre los indigenas 'QOM' (tobas), en: Centro Interdisciplinario de ciencias etnolinguísticas y antropológico-sociales. Rosario, Facultad de Humanidades y Arte UNR, 1998.

VIGLIANCHINO, M. «En torno a la división social del trabajo. Apuntes sobre las mujeres tobas migrantes», en: Revista de la escuela de Antropología. Vol. IV, Rosario, UNR, 1998.

Publicación de la Secretaría de Salud Pública de la Municipalidad de Rosario. «Atención Primaria de la Salud». Rosario, Dirección General de Comunicación Social, 2000. 